\title{
Simulation of whistler mode propagation for low latitude stations
}

\author{
Kalpana Singh ${ }^{1}$, R. P. Singh ${ }^{1}$, and Orsolya E. Ferencz ${ }^{2}$ \\ ${ }^{1}$ Atmospheric Research laboratory, Department of Physics, Banaras Hindu University, Varanasi, India-221 005 \\ ${ }^{2}$ Department of Geophysics, Eotvos University, Budapest, H-1117, Hungary
}

(Received March 15, 2004; Revised June 20, 2004; Accepted July 22, 2004)

\begin{abstract}
Representing lightning discharge current source by a Dirac delta function, Maxwell's equations are solved to derive the expression for wave-electric field as a function of frequency and distance including the effect of interparticle collisions. The exact time-dependence of the propagating non-monochromatic signal for the realistic magnetospheric model is computed for low latitude stations (in India). The computation is extended for the wave propagating through different regions of the magnetosphere and results are compared with the measured data. Points of agreements and differences are illuminated.
\end{abstract}

Key words: Simulation, magnetoplasma, whistler, dynamic spectra.

\section{Introduction}

The return stroke of lightning discharge is known to generate electromagnetic signal in wide frequency band in all direction. Part of the signal energy is reflected back from the ionosphere and propagate in the space between the earth and the ionosphere. These waves propagate to long distances without appreciable dispersion and attenuation. These signals received on the earth's surface are known as sferics. Part of the signal penetrates the ionosphere and propagates along the geomagnetic field lines in whistler mode with almost very little attenuation. At mid and high latitudes ducts formed by the electron density fluctuations support ducted mode propagation of whistler mode signal with almost negligible attenuation (Helliwell, 1965; Hayakawa et al., 1986). The supporting duct requires electron density fluctuation $\sim 5-10 \%$ at mid and high latitudes. At very low latitudes, for the ducted propagation required electron density fluctuation is few hundred percent (Singh and Tantry, 1973; Hasegawa et al., 1978). There is no physical mechanism, which can support such a high magnitude of fluctuation. Further, geomagnetic field lines at low latitudes are quite curved. Under these diverse conditions, ducted mode propagation of VLF waves at low latitudes is ruled out and non-ducted or pro-longitudinal mode of propagation is considered (Singh, 1993). Two dimensional ray-tracing computation studies suggested non-ducted propagation at these low latitudes (Liang et al., 1985; Thomson, 1987; Ohta et al., 1992). These computational results showed that a preferred non-ducted propagation channel exists in the geomagnetic latitude range $10^{\circ}-15^{\circ}$. Ohta et al. (1997) considered threedimensional ray-tracing computations for the realistic magnetospheric model and showed that waves with wave-normal angles $\sim 3^{\circ}$ entering into the ionosphere/magnetosphere and propagating in the non-ducted mode could reach the con-

Copy right (c) The Society of Geomagnetism and Earth, Planetary and Space Sciences (SGEPSS); The Seismological Society of Japan; The Volcanological Society of Japan; The Geodetic Society of Japan; The Japanese Society for Planetary Sciences; TERRAPUB. jugate points. They have shown that signal entering into the southern hemisphere could be received as echo-train whistlers. One and three hop whistlers with the dispersion 1:3 in the northern hemisphere at very low latitudes by means of both the ionospheric and ground reflection mechanisms could be received and such whistlers form echo-train whistlers. Keeping in view this limitation, our aim is to determine the space-time dependence of whistler mode signal as exact as possible for low latitude stations so that proper propagation mechanism can be illuminated.

Ferencz (1994) obtained one-dimensional whistler-mode solution of Maxwell's equations and used it in the interpretation of the characteristic features of the observed whistlers. The transient response to impulse or step like disturbances under the magnetohydrodynamic conditions were simulated to explain the characteristic features of geomagnetic field pulsations (Itonaga et al., 1997a, b). The waveforms due to these disturbances were directly simulated by a numerical inversion of the Laplace transform with orthonormal Laguerre functions. In order to explain optical emissions during thunderstorm, space and time evolution of the lightning generated electromagnetic pulse was studied using a two-dimensional numerical simulation (Rowland et al., 1995; Inan et al., 1996; Veronis et al., 1999). Cho and Rycroft (2001) developed a three dimensional code to calculate the optical emissions created by the electromagnetic pulse from a horizontal cloud-to-cloud discharge. These numerical simulations are based on the finite difference time domain treatment of Maxwell's equations. Recently, Nagano et al. (2003), using full wave analysis, have studied the ionospheric propagation of the lightning generated electromagnetic pulse with a model including a horizontally stratified ionosphere, free space and ground. Fourier spectral analysis and the plane wave expansion technique have been used.

In this paper we present the application of full-wave (Ferencz, 1994) whistler mode signal derived from Maxwell's equations propagating through a one-dimensional inhomogeneous medium. The magnetosphere is supposed to be weakly 


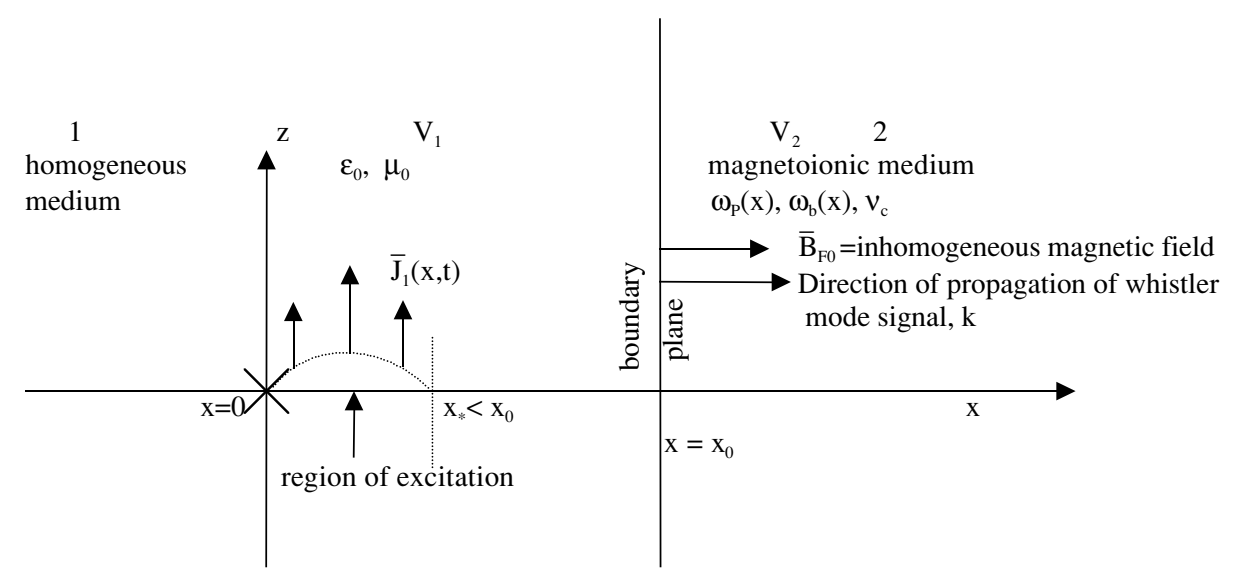

Fig. 1. Schematic model used in the computation of whistler mode wave propagating through and inhomogeneous magnetoplasma.

ionized inhomogeneous medium in which wave energy dispersion is caused by interaction between electrons and wave fields and dissipation is caused by collisions between electrons and neutrals. Solving Maxwell's equations using the Laplace transform, we derive wave field expression. Considering the space dependent electron density and ambient magnetic field, simulation technique has been developed to evaluate the wave field as a function of space and time. The results are used to explain some characteristic features of whistlers at low latitudes.

\section{Theoretical Formulation and Computational Results}

The source of electromagnetic signal is considered to be lightning discharge between cloud to ground, cloud to cloud, or intra-cloud. Cloud is generally located between 5-15 $\mathrm{kms}$ height above the ground surface, which is independent of plasma particles. Figure 1 shows the calculation model in which region 1 is considered to be the region between the surface of the earth and lower part of the ionosphere. Region 1 contains the excitation. Region 2 contains the plasma. Magnetic field is embedded everywhere. Let us consider magnetic field and plasma density to vary in the $x$ direction making gyro-frequency and plasma frequency space dependent. In this representation, medium ' 1 ' has no significant interaction with the signal, however the excitation appears inside of half-space ' 1 ', as shown in Fig. 1, in the time interval $0<t<t_{0}$ and in the $0 \leq x \leq x_{*}$ space interval, where $x_{*} \leq x_{0}$, and $x_{0}$ is the boundary surface separating the free space and magnetized medium. Let the direction of excitation, i.e. the direction of source current density be parallel to the $z$-axis, i.e., $\bar{J}_{1}(x, t)=J_{0}(x, t) \hat{e}_{z}$. The medium ' 2 ' is supposed to be an infinite half space i.e., the magnetosphere. The change in the direction of $\bar{B}_{F 0}$ is however neglected.

In this computation we have considered whistler mode propagation along the magnetic field direction, as ducted mode propagation at low latitude is not possible. The lightning discharge radiates electromagnetic signal in all direction. Only a small portion of radiated energy can penetrate the ionosphere along the geomagnetic field line depending upon the orientation of lightning discharge, its location, the strength of return stroke current etc. Because of this rea- son, only small number of whistler is observed at low latitudes, when intense discharges are properly oriented. Further, whistler producing lightning discharges should have dynamic spectra with maximum amplitude in the frequency range 5-10 kHz. In view of ray-tracing results (Ohta et al., 1997) we may consider non-ducted propagation along the field line. Time and space development of electromagnetic signal in any medium is governed by the Maxwell's equations. The interaction of signal with the medium is governed by the equation of motion and continuity equation. All these equations form a closed set of equations to describe the evolution of signal and its impact on medium through which it propagates. These equations are solved to obtain the expression for the signal as a function of space and time (see Appendix). The whistler-mode signal in a weakly inhomogeneous lossy ionosphere/magnetosphere is obtained as

$$
\begin{aligned}
E_{2 z \omega}(x, t)= & -\frac{Z_{0}}{4 \pi} \int_{\omega_{\min }}^{\omega_{\max }} I_{x 0}(\omega) \sqrt{\frac{K_{1}(x, \omega}{K_{1}\left(x_{0}, \omega\right)}} \\
& \times \frac{k_{0}(\omega)}{K_{0}(\omega)+K_{1}\left(x_{0}, \omega\right)} \\
& \times e^{j\left[\omega t-\int_{x_{0}}^{x} K_{1}(\xi, \omega) d \xi\right]} d \omega \\
H_{2 y \omega}(x, t)= & \frac{1}{4 \pi} \int_{\omega_{\min }}^{\omega_{\max }} I_{x 0}(\omega) \sqrt{\frac{K_{1}(x, \omega)}{K_{1}\left(x_{0}, \omega\right)}} \\
& \times \frac{K_{1}\left(x_{0}, \omega\right)}{k_{0}(\omega)+K_{1}\left(x_{0}, \omega\right)} \\
& \times e^{j\left[\omega t-\int_{x_{0}}^{x} K_{1}(\xi, \omega) d \xi\right]} d \omega
\end{aligned}
$$

where, $k_{0}=\omega / c, I_{x 0}(\omega)$ is the amplitude of excitation signal and $K_{1}(x, \omega)=k_{1}(x, \omega) \eta_{1}(x, \omega)$, while $k_{1}(x, \omega)=$ $\frac{1}{c} \sqrt{\frac{\omega \omega_{b}(x) \omega_{p}^{2}(x)+\omega^{2}\left(\omega_{p}^{2}(x)+\omega_{b}^{2}(x)-\omega^{2}\right)}{\left(\omega_{b}^{2}(x)-\omega^{2}\right)}}$

$$
\begin{aligned}
& \eta_{1}(x, \omega)=\sqrt{1+\frac{v_{C}^{2}}{2}\left[M_{1}(\omega)-N_{1}(\omega)\right]} \\
& \times\left\{1-j \frac{1}{2} v_{C}\left[\phi_{1}(\omega)+\phi_{2}(\omega)\right]\right\}, \\
& M_{1}(\omega)= \\
& \frac{\left[2 \omega Q(\omega)+\omega_{p}^{2}(x) \omega_{b}(x)\right]^{2}-2 Q(\omega)\left[\omega^{2} F(\omega)+\omega_{p}^{2}(x) \omega_{b}(x) \omega\right]}{\left[\omega^{2} F(\omega)+\omega_{p}^{2}(x) \omega_{b}(x) \omega\right]^{2}}
\end{aligned}
$$


(a)

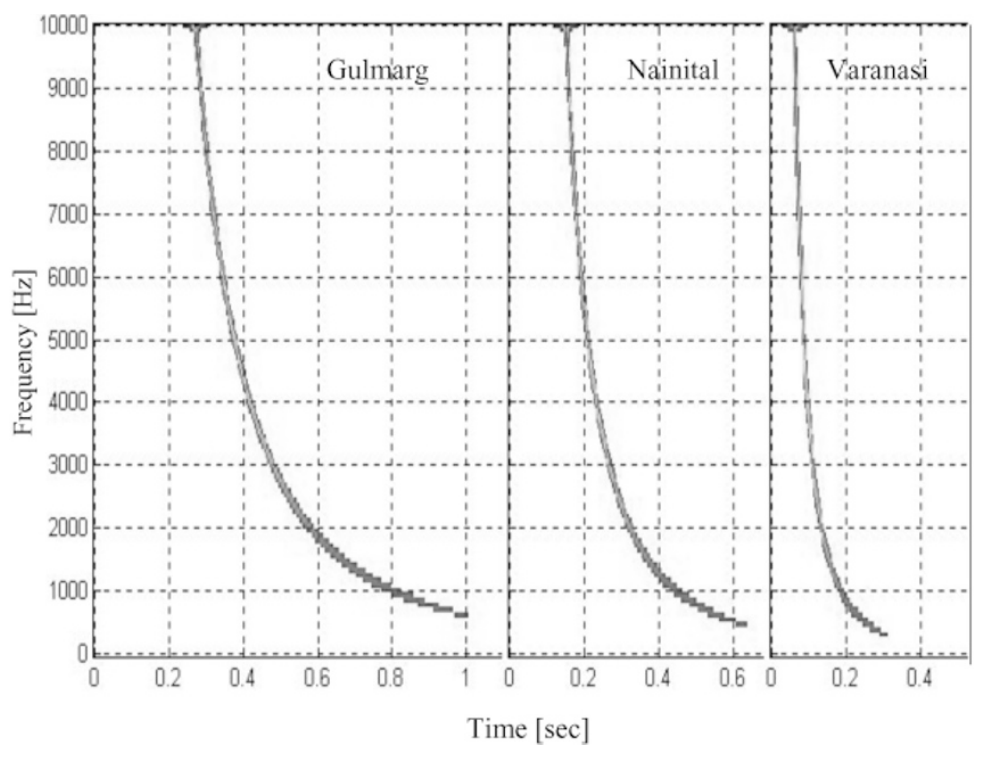

(b)

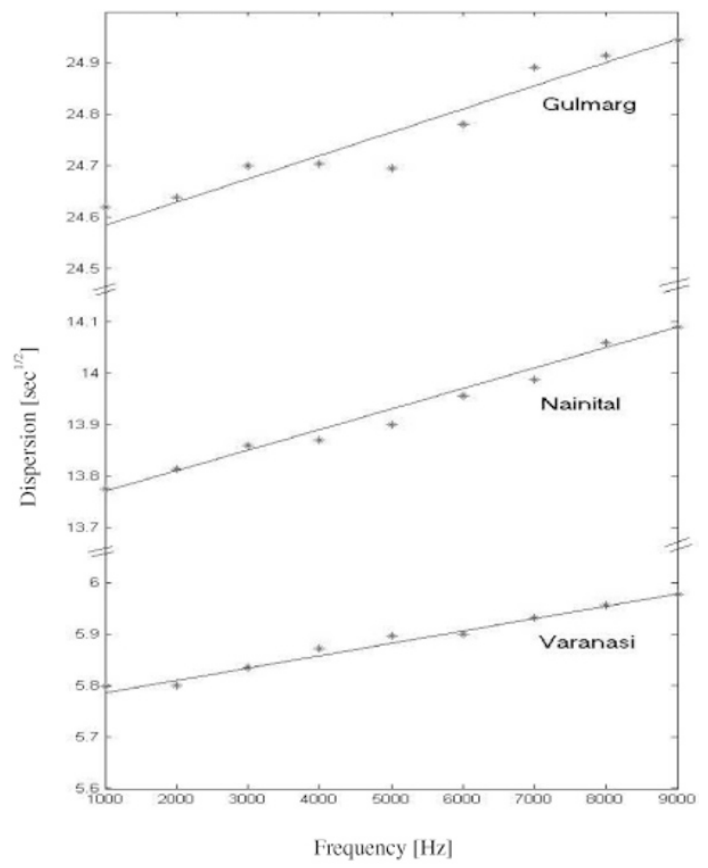

Fig. 2. (a) Dynamic spectra of whistler mode signal simulated for Varanasi, Nainital and Gulmarg. (b) Variation of dispersion as a function of frequency corresponding to above stations.

$$
\begin{aligned}
& \text { where } Q(\omega)=\omega_{p}^{2}(x)-\omega^{2}, F(\omega)=\omega_{p}^{2}(x)+\omega_{b}^{2}(x)-\omega^{2} \\
& \qquad N_{1}(\omega)=\frac{2\left(\omega_{b}^{2}(x)+\omega^{2}\right)}{\left(\omega_{b}^{2}(x)-\omega^{2}\right)^{2}} \\
& \phi_{1}(\omega)=\frac{2 \omega Q(\omega)+\omega_{b}(x) \omega_{p}^{2}(x)}{\omega^{2} F(\omega)+\omega_{b}(x) \omega_{p}^{2}(x) \omega-v_{C}^{2} Q(\omega)} \\
& \text { and } \\
& \qquad \phi_{2}(\omega)=\frac{2 \omega}{\left(\omega_{p}^{2}(x)-\omega^{2}\right)+v_{C}^{2}} .
\end{aligned}
$$

From the above equations all the further field components can be determined. The commonly known whistler spectrum pattern can be obtained from this space time function at a given space by the Fast Fourier transform method used in signal processing.

Time-frequency involves mapping a signal (i.e., a onedimensional function of time) into an image (i.e., a two dimensional function of time and frequency) that displays the temporal localization of the signal's spectral components. Moreover, the Short Time Fourier Transform (STFT) represents a sort of compromise between the time-and frequency-based views of a signal. It provides some information about both when and at what frequencies a signal event occur, although this information can be only obtained with limited precision, and that precision is determined by the size of the window which is governed by uncertainty principle.

Procedure of STFT involves the chopping of signal into short pieces and then fast Fourier transform is taken piecewise,

$$
S T F T=\int_{-\infty}^{+\infty} x(\tau) W^{*}(\tau-t) e^{-j \omega \tau} d \tau
$$

where $x(\tau)$ is discrete time samples of signal and $W^{*}(t)$ is the complex conjugate of window function, these window functions are applied to avoid discontinuities at the end of a set of data. The smaller these discontinuities are, the faster the side slopes drop. We have used Hamming window which is the most common in speech analysis.

Equations (1) and (2) are used to compute the variations of wave-amplitude as a function of space and time. In the numerical computation excitation source is considered to be Dirac delta distribution. The transfer function characterises the propagational features of the wave through the medium. The amplitude-time function of the whistler mode signal is directly obtained from the closed-form solution of the above equations. The dynamic spectrum of the signal can be calculated from the time-function by one of the well-known signal processing procedure and common FFT algorithms. In the numerical computation, we have considered dipole nature of geomagnetic field variation and plasma density distribution along the path of propagation is taken as diffusive equilibrium model DE-1 proposed by Park (1972). In particular, we have considered the plasma consisting of electrons, oxygen ions, hydrogen ions and helium ions. The distribution of ions is taken as $\mathrm{N}\left(\mathrm{O}^{+}\right)=90 \%, \mathrm{~N}\left(\mathrm{He}^{+}\right)=2 \%$ and $\mathrm{N}\left(\mathrm{H}^{+}\right)=8 \%$. In diffusive equilibrium model for $N(x), x$ is the arc-length along the magnetic line of force measured from the reference height $x_{0}$.

Figure 2(a) shows the dynamic spectra of whistlers computed from full-wave model for (i) Varanasi ( $L=1.07)$, (ii) Nainital $(L=1.17)$ and (iii) Gulmarg $(L=1.28)$ respectively. The dispersion of the wave depends upon path length, magnetic field and electron density distribution along the path of propagation. Hence it varies from station to station. The zero frequency dispersion of the computed spectrum and shown in Fig. 2(b) is 5.79, 13.78 and $24.58 \mathrm{sec}^{\frac{1}{2}}$ 


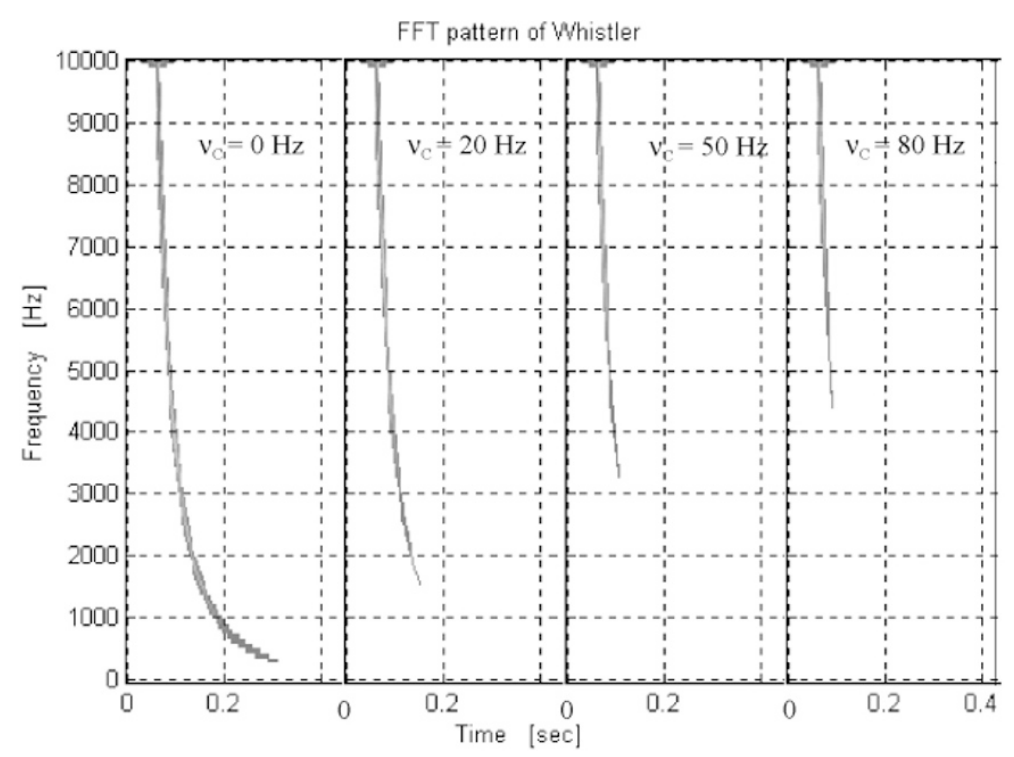

Fig. 3. FFT spectrum of computed whistlers for Varanasi with different interparticle collision frequency.

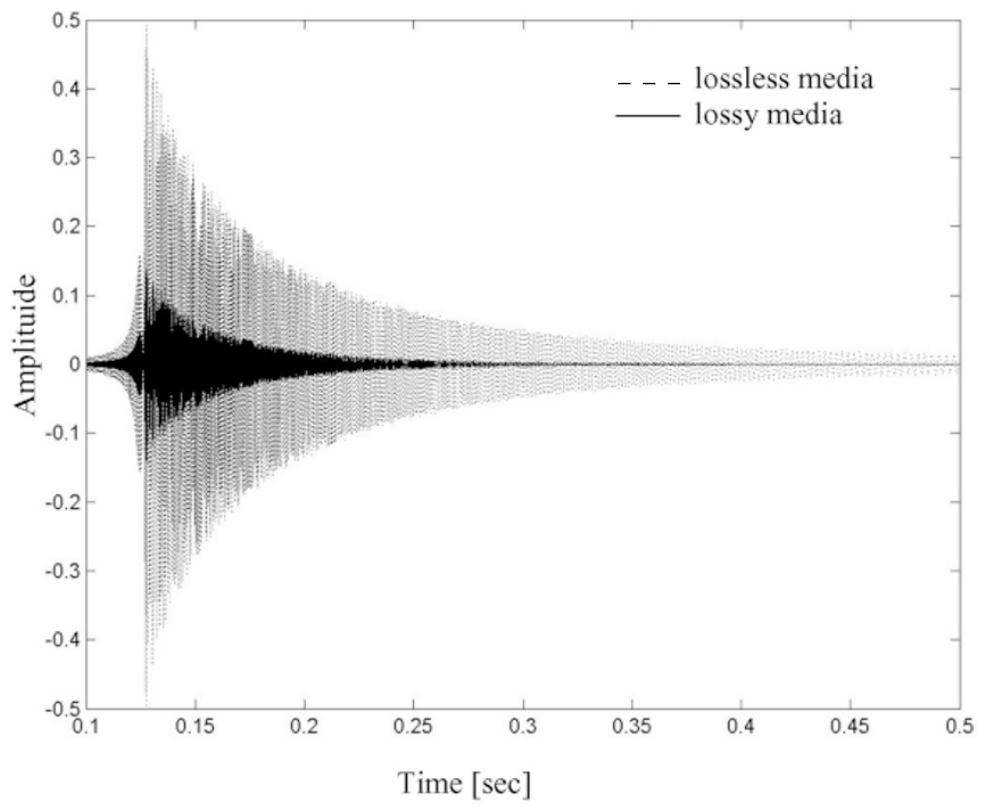

Fig. 4. Detailed amplitude time-functions of computed-whistlers $\left(v_{C}=0 \mathrm{~Hz}\right.$ and $\left.v_{C}=10 \mathrm{~Hz}\right)$.

corresponding to Varanasi, Nainital and Gulmarg respectively. We found that these whistlers follow Eckersley law (Eckersley, 1935). The dispersion of observed whistlers at Varanasi is $\sim 12 \sec ^{\frac{1}{2}}$ (Singh et al., 1998). Dispersion of recorded whistlers at Nainital and Gulmarg is 19 and $24 \mathrm{sec}^{\frac{1}{2}}$ respectively (Somayajulu et al., 1972). If correction is made for the ionosphere path of propagation (Park, 1972; Singh et al., 1993) then the observed dispersion value reduces to 5, 11 and $17 \sec ^{\frac{1}{2}}$ for Varanasi, Nainital and Gulmarg respectively. This shows that the simulated whistlers resemble very closely with those observed at the low latitude Indian stations. In these computations we have considered whistler wave propagation exactly parallel to the magnetic field line. If we change the model of charge particle distribution then zero frequency dispersion also changes.

The dynamic spectra shown in Fig. 3 shows the simulated results for lossless as well as lossy medium for Varanasi. Instead of height dependent collisions we have considered different constant collision frequencies. Figure 3 is dynamic spectra corresponding to Varanasi for $v_{c}=20,50$ and $80 \mathrm{~Hz}$. Thus, the lower frequency part of the spectrum disappears showing that the effect of collision is not equally strong for whole frequency range considered. From Fig. 3 we note that when collision frequency becomes appreciable then at low latitudes whistler wave would appear as sferics with attenuation of lower frequencies. Thus whistlers propagating during disturbed conditions at low latitudes when collisions become dominant may appear as sferics. Such signal require full wave analysis. Enlarged, detailed pattern of whistler-mode signal as a function of time for lossless and lossy model (for a typical collision frequency $v_{c}=10 \mathrm{~Hz}$ ) is shown in Fig. 4 . It is important to note that the zero level is not shifted in lossy signal, only the amplitude is decreased. An increase in colli- 


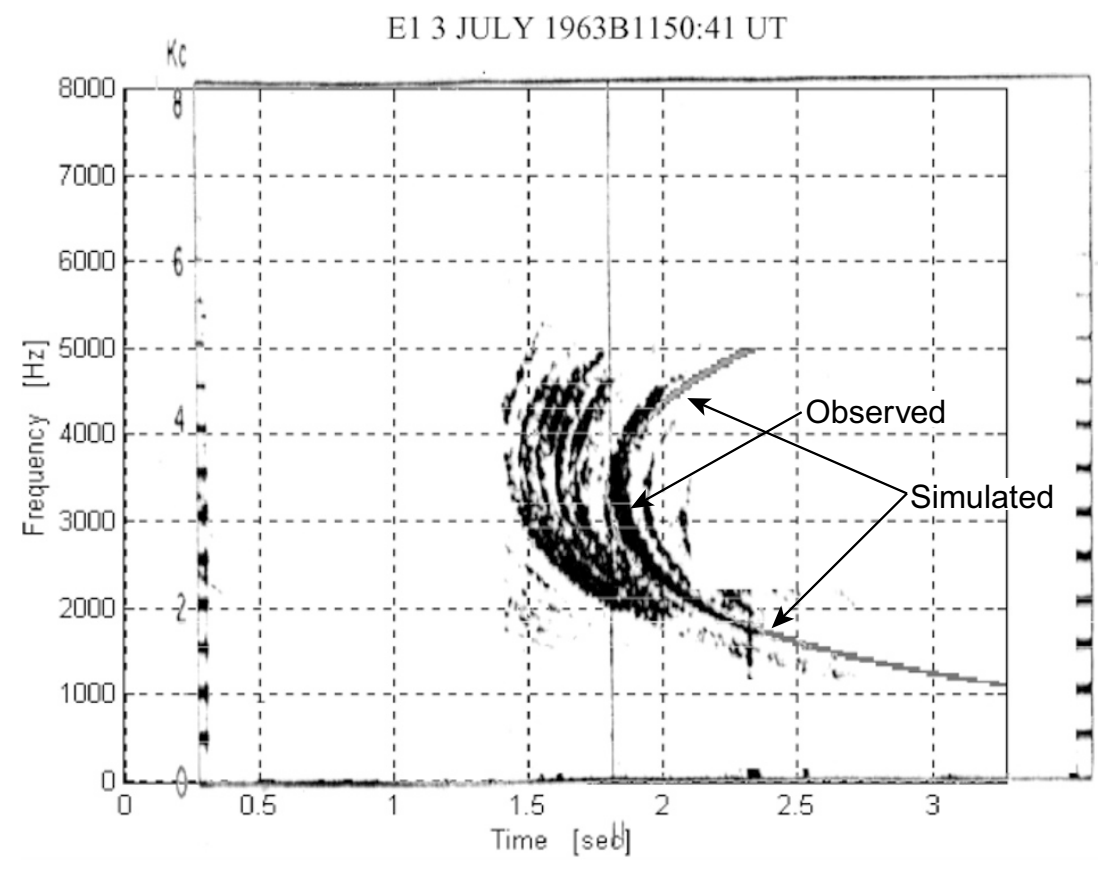

Fig. 5. Simulation of nose whistler (3 July 1963, 1150:41 UT, Eights Station, Antarctica (Guthart, 1965)). Observed and simulated are marked by arrow.

sion frequency causes strong decrease in amplitude. Computation has been made for different collision frequencies but the same is not shown in the figure. Amplitude decreases with time, because the volume of the space for observation of the signal increases with time while the poynting vector of the signal remains constant (Ferencz, 1994). Therefore, amplitude of the signal has to decrease with time even in a lossless medium, but if losses are also included in the calculation then amplitude of signal gets decreased as the wave propagates in space because of the attenuation of the wave. Thus, the wave amplitude in lossy medium decreases faster than that in the lossless medium.

As an example, we have considered whistlers recorded at mid latitude station Eights station, Antartica (Geom. Lat. = $\left.64^{\circ} \mathrm{S}, L=5.2037\right)$ on July 3, 1963 which is shown in Fig. 5 (Guthart, 1965). The propagation path is considered as $L=4.59$. Considering the parameters $T_{e}=5.7 \times 10^{4} \mathrm{~K}$, $n_{e}=1.85 \times 10^{3} / \mathrm{cm}^{3}$ and $h_{\text {ref }}=1 \times 10^{3} \mathrm{~km}$ where $h_{\text {ref }}$ is the height that is assumed to be the altitude of the penetration of signal into the magnetosphere and $T_{e}$ and $n_{e}$ are the respective temperature and number density at reference height, we have simulated whistler for Dirac-delta excitation. FFT spectra of this modeled whistler is also shown in Fig. 5. Comparing simulated and the observed whistlers, we find that the computational technique reported in this paper could precisely reproduce nose whistler also. Therefore, this technique can be used to reproduce the nose frequency of VLF signals at low latitudes, which could not be detected experimentally. In the absence of nose frequency path of propagation is determined with certain approximation by using extension method (Sazhin et al., 1992; Singh et al., 1998).

In this report we have presented modeling of observed whistlers at low latitudes by solving Maxwell's equations.
The method developed is useful in analyzing whistlers at low latitudes and in exploiting the dynamic spectra to derive the medium parameters with better accuracy and reliability.

Acknowledgments. One of the authors (K. S.) is thankful to Council of Scientific and Industrial Research, New Delhi for providing financial support.

\section{Appendix.}

The interaction of signal with the plasma medium is governed by the equation of motion and Maxwell's equations, which form a close set of equations. The charged particle's velocity $\bar{v}$ is governed by the momentum equation

$$
m \frac{d \bar{v}}{d t}+m v_{c} \bar{v}=q\left(\bar{E}+\bar{v} \times \bar{B}_{F 0}\right)
$$

where $B=B_{F 0}+B_{1}(r, t), B_{F 0}$ is steady state magnetic field taken to be in the $x$ direction, $E(r, t)$ and $B_{1}(r, t)$ are electric and magnetic fields of the wave. In general, $B_{1} \ll B_{F 0}$ and hence it is neglected. Writing Eq. (1) in component form and following the procedure of Kamke $(1956,1965)$ we obtain solution in the following form

$$
\begin{aligned}
v_{x}= & e^{-v_{c} t}\left[C+\int_{t} \frac{q}{m} E_{x}(\tau) e^{v_{c} \tau} d \tau\right] \\
v_{y}= & e^{-v_{c} t}\left(C_{1} \cos \omega_{b} t+C_{2} \sin \omega_{b} t\right) \\
& +\frac{1}{\omega_{b}} \int_{t} \varphi_{y}(\tau) e^{-v_{c}(t-\tau)} \sin \omega_{b}(t-\tau) d \tau \\
v_{z}= & e^{-v_{c} t}\left(C_{1} \cos \omega_{b} t+C_{2} \sin \omega_{b} t\right) \\
& +\frac{1}{\omega_{b}} \int_{t} \varphi_{z}(\tau) e^{-v_{c}(t-\tau)} \sin \omega_{b}(t-\tau) d \tau
\end{aligned}
$$

where

$$
\varphi_{y}(\tau)=\frac{q}{m}\left[E_{y}^{\prime}(\tau)+v_{c} E_{y}(\tau)-\omega_{b} E_{z}(\tau)\right]
$$




$$
\varphi_{z}(\tau)=\frac{q}{m}\left[E_{z}^{\prime}(\tau)+v_{c} E_{z}(\tau)+\omega_{b} E_{y}(\tau)\right]
$$

The homogeneous terms of above equations do not propagate, as they have no contribution to the excitation and they are not real signals, therefore these parts will be eliminated in the computation of current, which is written as

$$
\begin{aligned}
J_{x}= & q N v_{x}=\varepsilon_{0} \omega_{p}^{2} e^{-v_{c} t}\left[\int_{t} E_{x}(\bar{r}, \tau) e^{v_{c} \tau} d \tau\right] \\
J_{y}= & \varepsilon_{0} \omega_{p}^{2}\left\{\frac{1}{\omega_{b}} \int_{t} \frac{\partial E_{y}}{\partial \tau} e^{-v_{c}(t-\tau)} \sin \omega_{b}(t-\tau) d \tau\right. \\
& +\frac{1}{\omega_{b}} v_{c} \int_{t} E_{y} e^{-v_{c}(t-\tau)} \sin \omega_{b}(t-\tau) d \tau \\
& \left.-\int_{t} E_{z} e^{-v_{c}(t-\tau)} \sin \omega_{b}(t-\tau) d \tau\right\} \\
J_{z}= & \varepsilon_{0} \omega_{p}^{2}\left\{\frac{1}{\omega_{b}} \int_{t} \frac{\partial E_{z}}{\partial \tau} e^{-v_{c}(t-\tau)} \sin \omega_{b}(t-\tau) d \tau\right. \\
& +\frac{1}{\omega_{b}} v_{c} \int_{t} E_{z} e^{-v_{c}(t-\tau)} \sin \omega_{b}(t-\tau) d \tau \\
& \left.+\int_{t} E_{y} e^{-v_{c}(t-\tau)} \sin \omega_{b}(t-\tau) d \tau\right\} .
\end{aligned}
$$

Substituting above equations into Maxwell's equations

$$
\bar{\nabla} \times \bar{H}=\bar{J}+\varepsilon_{0} \frac{\partial \bar{E}}{\partial t} ; \quad \text { and } \quad \bar{\nabla} \times \bar{E}=-\mu_{0} \frac{\partial \bar{H}}{\partial t}
$$

We obtain the following differential equations

$$
\left.\begin{array}{l}
\frac{\partial H_{z}}{\partial y}-\frac{\partial H_{y}}{\partial z} \\
=\varepsilon_{0}\left\{\omega_{p}^{2} e^{-v_{c} t} \int_{t} E_{x}(\bar{r}, \tau) e^{v_{c} \tau} d \tau+\frac{\partial E_{x}}{\partial t}\right\} \\
\frac{\partial H_{x}}{\partial z}-\frac{\partial H_{z}}{\partial x} \\
=\varepsilon_{0}\left\{\frac{\omega_{p}^{2}}{\omega_{b}} \int_{t} \frac{\partial E_{y}}{\partial \tau} e^{-v_{c}(t-\tau)} \sin \omega_{b}(t-\tau) d \tau\right. \\
+\frac{\omega_{p}^{2}}{\omega_{b}} v_{c} \int_{t} E_{y} e^{-v_{c}(t-\tau)} \sin \omega_{b}(t-\tau) d \tau \\
\left.-\omega_{p}^{2} \int_{t} E_{z} e^{-v_{c}(t-\tau)} \sin \omega_{b}(t-\tau) d \tau+\frac{\partial E_{y}}{\partial t}\right\} \\
\frac{\partial H_{y}}{\partial x}-\frac{\partial H_{x}}{\partial y} \\
=\varepsilon_{0}\left\{\frac{\omega_{p}^{2}}{\omega_{b}} \int_{t} \frac{\partial E_{z}}{\partial \tau} e^{-v_{c}(t-\tau)} \sin \omega_{b}(t-\tau) d \tau\right. \\
+\frac{\omega_{p}^{2}}{\omega_{b}} v_{c} \int_{t} E_{y} e^{-v_{c}(t-\tau)} \sin \omega_{b}(t-\tau) d \tau \\
\left.+\omega_{p}^{2} \int_{t} E_{y} e^{-v_{c}(t-\tau)} \sin \omega_{b}(t-\tau) d \tau+\frac{\partial E_{z}}{\partial t}\right\} \\
\frac{\partial E_{z}}{\partial y}-\frac{\partial E_{y}}{\partial z}=-\mu_{0} \frac{\partial H_{x}}{\partial t} \\
\frac{\partial E_{x}}{\partial z}-\frac{\partial E_{z}}{\partial x}=-\mu_{0} \frac{\partial H_{y}}{\partial t} \\
\frac{\partial E_{y}}{\partial x}-\frac{\partial E_{x}}{\partial y}=-\mu_{0} \frac{\partial H_{z}}{\partial t} .
\end{array}\right\}
$$

Taking time derivative of Eqs. (4a) and using Eqs. (4b), we obtain

$$
\frac{1}{c^{2}}\left(\omega_{p}^{2} E_{x}+\frac{\partial^{2} E_{x}}{\partial t^{2}}\right)=0 .
$$

Equation (5a) describes plasma oscillation and hence for a plane wave signal propagating in $x$-direction, we consider $E_{x} \equiv 0$, and $\frac{\partial}{\partial y}=\frac{\partial}{\partial z} \equiv 0$.

The remaining electric field components are obtained as

$$
\begin{aligned}
\frac{\partial^{2} E_{y}}{\partial x^{2}} & =\frac{1}{c^{2}}\left\{\omega_{p}^{2}(x) \int_{0}^{t} \frac{\partial E_{y}}{\partial \tau} e^{-v_{c}(t-\tau)} \cos \omega_{b}(x)(t-\tau) d \tau\right. \\
& +v_{c} \omega_{p}^{2}(x) \int_{0}^{t} E_{y}(\tau) e^{-v_{c}(t-\tau)} \cos \omega_{b}(x)(t-\tau) d \tau \\
& -\omega_{b}(x) \omega_{p}^{2}(x) \int_{0}^{t} E_{z}(\tau) e^{-v_{c}(t-\tau)} \cos \omega_{b}(x)(t-\tau) d \tau \\
& \left.+\frac{\partial^{2} E_{y}}{\partial t^{2}}\right\} \\
\frac{\partial^{2} E_{z}}{\partial x^{2}} & =\frac{1}{c^{2}}\left\{\omega_{p}^{2}(x) \int_{0}^{t} \frac{\partial E_{z}}{\partial \tau} e^{-v_{c}(t-\tau)} \cos \omega_{b}(x)(t-\tau) d \tau\right. \\
+ & v_{c} \omega_{p}^{2}(x) \int_{0}^{t} E_{z}(\tau) e^{-v_{c}(t-\tau)} \cos \omega_{b}(x)(t-\tau) d \tau \\
+ & +\omega_{b}(x) \omega_{p}^{2}(x) \int_{0}^{t} E_{y}(\tau) e^{-v_{c}(t-\tau)} \cos \omega_{b}(x)(t-\tau) d \tau \\
+ & \left.\frac{\partial^{2} E_{z}}{\partial t^{2}}\right\} .
\end{aligned}
$$

Using Laplace transforms in space and time can solve the above equations. In the present case plasma parameters depend on space and hence Laplace transformation in time will be only considered. As for longitudinal case is considered, the W. K. B. solution will be used to solve for the field components (Ferencz, 1977), keeping in mind that now the signal is non-monochromatic.

In this coordinate system shown in Fig. 1 the planepolarized excitation will have zero initial values along $y$ axis at the boundary surface. By choosing suitable $t=0$ moment - the space depending initial values along $z$-axis will also be zero.

$$
\left.\begin{array}{l}
E_{i}(x=0, t)=e_{i 0 t}(x) \leftrightarrow e_{i 0 t}(s), \\
E_{i}(x, t=0)=e_{i x 0}(t) \leftrightarrow e_{i x 0}(p), \\
\left.\frac{\partial E_{i}(x, t)}{\partial x}\right|_{x=0}=e_{i 0 t}^{\prime}(t) \leftrightarrow e_{i 0 t}^{\prime}(s), \quad \text { and } \\
\left.\frac{\partial E_{i}(x, t)}{\partial t}\right|_{t=0}=\dot{e}_{i x 0}(x) \leftrightarrow \dot{e}_{i x 0}(p),
\end{array}\right\}
$$

where $i=y, z$.

Using the above boundary conditions and Laplace Transform rules, the transformed form of Eqs. (5b) and (5c) becomes

$$
\begin{aligned}
& c^{2} p^{2} E_{y}(p, s)=\left[\omega_{p}^{2}(x) \frac{\left(s+v_{c}\right)^{2}}{\left(s+v_{c}\right)^{2}+\omega_{b}^{2}(x)}+s^{2}\right] \\
& \times E_{y}(p, s) \\
& -\omega_{p}^{2}(x) \omega_{b}(x) \frac{\left(s+v_{c}\right)}{\left(s+v_{c}\right)^{2}+\omega_{b}^{2}(x)} E_{z}(p, s)
\end{aligned}
$$

and

$$
\begin{aligned}
& c^{2} p^{2} E_{z}(p, s)=\left[\omega_{p}^{2}(x) \frac{\left(s+v_{c}\right)^{2}}{\left(s+v_{c}\right)^{2}+\omega_{b}^{2}(x)}+s^{2}\right] \\
& \times E_{z}(p, s)+\omega_{p}^{2}(x) \omega_{b}(x) \frac{\left(s+v_{c}\right)}{\left(s+v_{c}\right)^{2}+\omega_{b}^{2}(x)} E_{y}(p, s) \\
& +c^{2}\left[p e_{z x 0}(s)+\dot{e}_{z x 0}(s)\right] .
\end{aligned}
$$


From Eqs. (7a) and (7b) and writing, $e_{z x 0}(s)=A(s)$ and $\dot{e}_{z x 0}(s)=B(s), u=s+v_{c}$, we obtain

$$
\begin{aligned}
& E_{y}(p, s) \\
& =c^{2} \frac{-\left(\omega_{b}(x) \omega_{p}^{2}(x) u\right) R(u)[p A(s)+B(s)]}{\left[c^{2} p^{2} R(u)-\omega_{p}^{2}(x) u^{2}-s^{2} R(u)\right]^{2}+\left[u \omega_{b}(x) \omega_{p}^{2}(x)\right]^{2}}
\end{aligned}
$$

where $R(u)=u^{2}+\omega_{b}^{2}(x)$ and

$$
\begin{aligned}
& E_{z}(p, s)=E_{y}(p, s) \\
& \times \frac{c^{2} p^{2}\left(u^{2}+\omega_{b}^{2}(x)\right)-\omega_{p}^{2}(x) u^{2}-s^{2}\left(u^{2}+\omega_{b}^{2}(x)\right)}{-\left(u \omega_{b}(x) \omega_{p}^{2}(x)\right)} .
\end{aligned}
$$

The above Eqs. can be rewritten in the following form,

$$
\begin{aligned}
E_{y}(p, s)= & \frac{c^{2} a_{0}(s) a_{0}^{\prime}(s) a_{2}(s)}{b_{3}(s)} \\
& \times \frac{p+\frac{a_{1}(s)}{a_{2}(s)}}{p^{4}-\frac{b_{2}(s)}{b_{3}(s)} p^{2}+\frac{b_{1}(s)}{b_{3}(s)}}
\end{aligned}
$$

and,

$$
\begin{aligned}
E_{z}(p, s)= & \frac{c^{2} a_{0}^{\prime}(s) a_{4}(s) a_{2}(s)}{b_{3}(s)} \\
& \times \frac{\left[p+\frac{a_{1}(s)}{a_{2}(s)}\right]\left[p^{2}-\frac{a_{3}(s)}{a_{4}(s)}\right]}{p^{4}-\frac{b_{2}(s)}{b_{3}(s)} p^{2}+\frac{b_{1}(s)}{b_{3}(s)}}
\end{aligned}
$$

where, $a_{0}(s)=-\omega_{b}(x) \omega_{p}^{2}(x) u, a_{0}^{\prime}(s)=\left(u^{2}+\omega_{b}^{2}(x)\right)$,

$$
\begin{aligned}
a_{1}(s)= & B(s), \quad a_{2}(s)=A(s), \\
a_{3}(s)= & \omega_{p}^{2}(x) u^{2}+s^{2}\left(u^{2}+\omega_{b}^{2}(x)\right), \\
a_{4}(s)= & \left(u^{2}+\omega_{b}^{2}(x)\right) c^{2}, \\
b_{1}(s)= & \left(u \omega_{b}(x) \omega_{p}^{2}(x)\right)^{2}+\omega_{p}^{4}(x) u^{4} \\
& +s^{4}\left(u^{2}+\omega_{b}^{2}(x)\right)^{2}+2 \omega_{p}^{2}(x) u^{2} s^{2}\left(u^{2}+\omega_{b}^{2}(x)\right), \\
b_{2}(s)= & 2 c^{2}\left(u^{2}+\omega_{b}^{2}(x)\right)\left[\omega_{p}^{2}(x) u^{2}+s^{2}\left(u^{2}+\omega_{b}^{2}(x)\right)\right], \\
b_{3}(s)= & c^{4}\left(u^{2}+\omega_{b}^{2}(x)\right)^{2} .
\end{aligned}
$$

Equations (9a) and (9b) show that each component of electric fields has four poles, which are given by

$$
\begin{aligned}
& p_{1,2,3,4}(x, s) \\
& = \pm \frac{1}{c} \sqrt{\frac{\omega_{p}^{2}(x) u^{2}+s^{2}\left[u^{2}+\omega_{b}^{2}(x)\right] \pm j \omega_{p}^{2}(x) \omega_{b}(x) u}{u^{2}+\omega_{b}^{2}(x)}}
\end{aligned}
$$

Substituting $s=j \omega$, where $\omega$ is wave frequency, we obtain,

$$
\begin{aligned}
& p_{1,2,3,4}= \pm \frac{1}{c} \\
& \sqrt{\frac{-\omega^{2} F(\omega) \mp \omega_{p}^{2}(x) \omega_{b}(x) \omega+Q(\omega)\left(v_{C}^{2}+2 j \omega v_{C}\right) \pm j \omega_{p}^{2}(x) \omega_{b}(x) v_{C}}{G(\omega)+2 j \omega v_{C}+v_{C}^{2}} .}
\end{aligned}
$$

where $F(\omega)=\omega_{p}^{2}(x)+\omega_{b}^{2}(x)-\omega^{2} ; Q(\omega)=\omega_{p}^{2}(x)-\omega^{2}$, and $G(\omega)=\omega_{b}^{2}-\omega^{2}$.

Rearranging and separating the real and imaginary, the approximate expression of the poles for the cases of low loss (i.e., small $v_{C}$ ) reduces to

$$
\left.\begin{array}{l}
p_{1,2}=\mp j k_{1}(\omega) \eta_{1}(\omega) \\
p_{3,4}=\mp j \alpha_{3}(\omega) \lambda_{3}(\omega)
\end{array}\right\}
$$

where, $k_{1}(\omega)=\frac{1}{c} \sqrt{\frac{\omega \omega_{b}(x) \omega_{p}^{2}(x)+\omega^{2}\left(\omega_{p}^{2}(x)+\omega_{b}^{2}(x)-\omega^{2}\right)}{\left(\omega_{b}^{2}(x)-\omega^{2}\right)}}$,

$$
\begin{aligned}
\eta_{1}(\omega)= & \sqrt{1+\frac{v_{C}^{2}}{2}\left[M_{1}(\omega)-N_{1}(\omega)\right]} \\
& \times\left\{1-j \frac{1}{2} v_{C}\left[\phi_{1}(\omega)+\phi_{2}(\omega)\right]\right\},
\end{aligned}
$$

$M_{1}(\omega)=$

$\frac{\left[2 \omega Q(\omega)+\omega_{p}^{2}(x) \omega_{b}(x)\right]^{2}-2 Q(\omega)\left[\omega^{2} F(\omega)+\omega_{p}^{2}(x) \omega_{b}(x) \omega\right]}{\left[\omega^{2} F(\omega)+\omega_{p}^{2}(x) \omega_{b}(x) \omega\right]^{2}}$

$$
N_{1}(\omega)=\frac{2\left(\omega_{b}^{2}(x)+\omega^{2}\right)}{\left(\omega_{b}^{2}(x)-\omega^{2}\right)^{2}}
$$

$$
\phi_{1}(\omega)=\frac{2 \omega Q(\omega)+\omega_{b}(x) \omega_{p}^{2}(x)}{\omega^{2} F(\omega)+\omega_{b}(x) \omega_{p}^{2}(x) \omega-v_{C}^{2}\left(\omega_{p}^{2}(x)-\omega\right)}
$$$$
\phi_{2}(\omega)=\frac{2 \omega}{\left(\omega_{p}^{2}(x)-\omega^{2}\right)+v_{C}^{2}} .
$$$$
\alpha_{3}(\omega)=\frac{1}{c} \sqrt{\frac{\omega \omega_{b}(x) \omega_{p}^{2}(x)-\omega^{2}\left(\omega_{p}^{2}(x)+\omega_{b}^{2}(x)-\omega^{2}\right)}{\left(\omega_{b}^{2}(x)-\omega^{2}\right)}},
$$

$$
\lambda_{3}(\omega)=\sqrt{1+\frac{v_{C}^{2}}{2}\left[M^{\prime}(\omega)-N^{\prime}(\omega)\right]}
$$

$$
\times\left\{1+j \frac{1}{2} v_{C}\left[\phi_{1}^{\prime}(\omega)-\phi_{2}^{\prime}(\omega)\right]\right\},
$$

$M_{1}^{\prime}(\omega)=\frac{2 Q(\omega) U(\omega)+\left[2 \omega Q(\omega)-\omega_{p}^{2}(x) \omega_{b}(x)\right]^{2}}{[U(\omega)]^{2}}$

where $U(\omega)=\omega \omega_{b}(x) \omega_{p}^{2}(x)+\omega^{4}-\omega^{2}\left(\omega_{p}^{2}(x)+\omega_{b}^{2}(x)\right)$

$$
\begin{aligned}
& N_{1}^{\prime}(\omega)=\frac{2\left(\omega_{b}^{2}(x)+\omega^{2}\right)}{\left(\omega_{b}^{2}(x)-\omega^{2}\right)^{2}}, \\
& \phi_{1}^{\prime}(\omega)=\frac{2 \omega Q(\omega)-V(x)}{\left(\omega V(x)+\omega^{4}\right)-\omega^{2}\left(\omega_{p}^{2}(x)+\omega_{b}^{2}(x)\right)+v_{C}^{2} Q(\omega)},
\end{aligned}
$$

where $V(x)=\omega_{b}(x) \omega_{p}^{2}(x)$, and

$$
\phi_{2}^{\prime}(\omega)=\frac{2 \omega}{\left(\omega_{p}^{2}(x)-\omega^{2}\right)+v_{C}^{2}}=\phi_{2}(\omega) \text {. }
$$

Hence the generalized solution is introduced, and, the homogeneous solution will be extended to the inhomogeneous case. So the poles are obtained for homogeneous case and are used further.

In the ELF and VLF range $0<\omega<\omega_{b}$ and $\omega_{b} \ll \omega_{p}$ for whistler mode propagation, poles $p_{2}$ and $p_{4}$ corresponds to backward propagating (reflected) signals and the remaining two modes relating $p_{1}$ and $p_{3}$ poles will be able to propagate in the forward direction.

Knowing the poles, the inverse Laplace-transformation of $E_{y}(p, s)$ and $E_{z}(p, s)$ is obtained, which yield spectra of the electric field components as a function of $x$.

$$
\begin{aligned}
E_{y}(x, \omega)= & \frac{c^{1} a_{0}(x, s) a_{0}{ }^{\prime}(x, s) a_{2}(s)}{b_{3}(x, s)} \\
& \times\left\{\frac{\left[p_{1}+\frac{a_{1}(s)}{a_{2}(s)}\right]}{\left[p_{1}^{2}-p_{3}^{2}\right]\left[p_{1}-p_{1}\right]} e^{\int_{0}^{x} p_{1} d x}\right. \\
& \left.-\frac{\left[-p_{1}+\frac{a_{1}(s)}{a_{2}(s)}\right]}{\left[p_{1}^{2}-p_{3}^{2}\right]\left[p_{1}-p_{2}\right]} e^{-\int_{0}^{x} p_{1} d x}\right\} \\
& =E_{y 1}(x, s)+E_{y 2}(x, s)
\end{aligned}
$$


and

$$
\begin{aligned}
E_{z}(x, \omega)= & \frac{c^{2} a_{0}{ }^{\prime}(x, s) a_{4}(x, s) a_{2}(s)}{b_{3}(x, s)} \\
& \times\left\{\frac{\left[p_{1}+\frac{a_{1}(s)}{a_{2}(s)}\right]\left[p_{1}^{2}-\frac{a_{3}(x, s)}{a_{4}(x, s)}\right]}{\left[p_{1}^{2}-p_{3}^{2}\right]\left[p_{1}-p_{2}\right]} e^{\int_{0}^{x} p_{1} d x}\right. \\
& \left.-\frac{\left[-p_{1}+\frac{a_{1}(s)}{a_{2}(s)}\right]\left[p_{1}^{2}-\frac{a_{3}(x, s)}{a_{4}(x, s)}\right]}{\left[p_{1}^{2}-p_{3}^{2}\right]\left[p_{1}-p_{2}\right]} e^{-\int_{0}^{x} p_{1} d x}\right\} \\
& =E_{z 1}(x, s)+E_{z 2}(x, s)
\end{aligned}
$$

where $p_{i}$ 's are function of $x$ and $s$.

As shown in Fig. 1, medium $z$ starts from $x=x_{0}$ and hence integration will be carried out from $x_{0}$ to $x$. Computing the polarization we find that $p_{1}$ and $p_{3}$ representing the right and left circularly polarized waves respectively. For whistler mode we consider only $p_{1}$ and corresponding field components are

$$
\left.\begin{array}{rl}
E_{y}(x, s)= & j \frac{1}{4 p_{1}(x, s)}\left\{\left[a_{2} p_{1}+a_{1}\right] e^{\int_{x_{0}}^{x} p_{1}(\xi, s) d \xi}\right. \\
& \left.-\left[-a_{2} p_{1}+a_{1}\right] e^{-\int_{x_{0}}^{2} p_{1}(\xi, s) d \xi}\right\} \\
E_{z}(x, s)= & \frac{1}{4 p_{1}(x, s)}\left\{\left[a_{2} p_{1}+a_{1}\right] e^{\int_{x_{0}}^{x} p_{1}(\xi, s) d \xi}\right. \\
& \left.-\left[-a_{2} p_{1}+a_{1}\right] e^{-\int_{x_{0}}^{x} p_{1}(\xi, s) d \xi}\right\}
\end{array}\right\}
$$

Substituting $s=j \omega$ and $p_{1}=-j k_{1}(\omega) \eta_{1}(\omega)=-j K_{1}(\omega)$, the field components, for propagation in forward direction, can be written as,

$$
\begin{aligned}
E_{z w}(x, \omega)= & \frac{1}{4 K_{1}(\omega)}\left[a_{2}(\omega) K_{1}(x, \omega)+j a_{1}(\omega)\right] \\
& \cdot e^{-j \int_{x_{0}}^{x} K_{1}(\xi, \omega) d \xi} \\
= & E_{z 0}^{+} e^{-j \int_{x_{0}}^{x} K_{1}(\xi, \omega) d \xi}
\end{aligned}
$$

and from Maxwell's equation,

$$
\begin{aligned}
H_{y w}(x, \omega) & =-\frac{K_{1}(x, \omega)}{\mu_{0} \omega} E_{z 0}^{+} e^{-j \int_{x_{0}}^{x} K_{1}(\xi, \omega) d \xi} \\
& =-\frac{E_{z 0}^{+}}{Z_{0}} n_{1}(x) e^{-j \int_{x_{0}}^{x} K_{1}(\xi, \omega) d \xi}
\end{aligned}
$$

where, $n_{1}(x)=\sqrt{\frac{\omega^{2}\left[\omega_{p}^{2}(x)+\omega_{b}^{2}(x)-\omega^{2}\right]+\omega \omega_{b}(x) \omega_{p}^{2}(x)}{\omega^{2}\left[\omega_{b}^{2}(x)-\omega^{2}\right]}} \eta_{1}(x, \omega)$, and suffix $w$ represents the whistler-mode. The condition to be satisfied for the spectral Poynting vector in a low loss medium $\left(v_{c} \ll \omega\right)$ is obtained as (Ferencz, 1994)

$$
S \sim\left(E_{z w}\right)\left(H_{y w}\right)^{*}=\left(E_{z 0}^{+}\right)^{2} \frac{n_{1}(x)}{Z_{0}} \equiv \text { const } \equiv\left(A_{0}^{+}\right)^{2} .
$$

Using Eq. (16), the field components are obtained as,

$$
\begin{aligned}
& E_{z w}(x, t)=\frac{1}{2 \pi} \int_{-\infty}^{+\infty} A_{0}^{+}(\omega) \\
& \times\left(\frac{\omega^{2}\left[\omega_{b}^{2}(x)-\omega^{2}\right]}{\omega^{2}\left[\omega_{p}^{2}(x)+\omega_{b}^{2}(x)-\omega^{2}\right]+\omega \omega_{b}(x) \omega_{p}^{2}(x)}\right)^{1 / 4} \\
& \times\left(\eta_{1}(x, \omega)\right)^{\frac{1}{2}} e^{j\left[\omega t-\int_{x_{0}}^{x} K_{1}(\xi, \omega) d \xi\right]} d \omega \\
& H_{y w}(x, t)=-\frac{1}{2 \pi Z_{0}} \int_{-\infty}^{+\infty} A_{0}^{+}(\omega)
\end{aligned}
$$

$$
\begin{aligned}
& \times\left(\frac{\omega^{2}\left[\omega_{b}^{2}(x)-\omega^{2}\right]}{\omega^{2}\left[\omega_{p}^{2}(x)+\omega_{b}^{2}(x)-\omega^{2}\right]+\omega \omega_{b}(x) \omega_{p}^{2}(x)}\right)^{1 / 4} \\
& \times\left(\eta_{1}(x, \omega)\right)^{1 / 2} e^{j\left[\omega t-\int_{x_{0}}^{x} K_{1}(\xi, \omega) d \xi\right]} d \omega
\end{aligned}
$$

where the unknown initial conditions characterizing the influence of the excitation are contained in $A_{0}^{+}(\omega)$.

Using the Method of Inhomogeneous Basic Modes (MIBM) (Ferencz, 1978) the coupling between the signals of medium " 1 " and " 2 " is represented by the initial conditions which is obtained as

$$
A_{0}^{+}(\omega)=\frac{-\frac{Z_{0}}{2} I_{x 0}(\omega)}{\sqrt[4]{\frac{\beta\left(x_{0}, \omega\right)}{\gamma\left(x_{0}, \omega\right)}} \frac{1}{\sqrt{\eta_{1}\left(x_{0}, \omega\right)}}+\sqrt[4]{\frac{\gamma\left(x_{0}, \omega\right)}{\beta\left(x_{0}, \omega\right)}} \sqrt{\eta_{1}\left(x_{0}, \omega\right)}}
$$

where

$$
\begin{aligned}
& \beta(x, \omega)=\omega^{2}\left[\omega_{b}^{2}(x)-\omega^{2}\right], \\
& \gamma(x, \omega)=\omega^{2}\left[\omega_{p}^{2}(x)+\omega_{b}^{2}(x)-\omega^{2}\right]+\omega \omega_{b}(x) \omega_{p}^{2}(x),
\end{aligned}
$$

$I_{x 0}(\omega)=I_{0}$ is the amplitude of excitation which is constant in the case of Dirac-delta excitation.

Substituting Eq. (18) into Eq. (17), the whistler-mode signal in weakly inhomogeneous lossy plasma is as follows

$$
\begin{aligned}
& E_{2 z \omega}(x, t)=-\frac{Z_{0}}{4 \pi} \int_{\omega_{\min }}^{\omega_{\max }} I_{x 0}(\omega) \\
& \times \sqrt{\frac{K_{1}\left(x_{0}, \omega\right)}{K_{1}(x, \omega)}} \frac{k_{0}(\omega)}{k_{0}(\omega)+K_{1}\left(x_{0}, \omega\right)} \\
& \times e^{j\left[\omega t-\int_{x_{0}}^{x} K_{1}(\xi, \omega) d \xi\right]} d \omega \\
& E_{2 y \omega}(x, t)=\frac{1}{4 \pi} \int_{\omega_{\min }}^{\omega_{\max }} I_{x 0}(\omega) \\
& \times \sqrt{\frac{K_{1}(x, \omega)}{K_{1}\left(x_{0}, \omega\right)}} \frac{K_{1}\left(x_{0}, \omega\right)}{k_{0}(\omega)+K_{1}\left(x_{0}, \omega\right)} \\
& \times e^{j\left[\omega t-\int_{x_{0}}^{x} K_{1}(\xi, \omega) d \xi\right]} d \omega
\end{aligned}
$$

where, $k_{0}=\omega / c$.

\section{References}

Cho, M. and M. J. Rycroft, Non-uniform ionization of the upper atmosphere due to the electromagnetic pulse from a horizontal lightning discharge, $J$. Atmos. Terr. Phys., 63, 559-580, 2001.

Eckersley, T. L., Musical atmospherics, Nature, 135, 104-105, 1935.

Ferencz, Cs., Electromagnetic wave propagation in inhomogeneous media: Strong and weak inhomogeneities, Acta Techn. Ac. Sci. H., 85(3-4), 433, 1977.

Ferencz, Cs., Electromagnetic wave propagation in inhomogeneous media: Method of inhomogeneous basic modes, Acta Techn. Ac. Sci. H., 86(1-2), 79, 1978.

Ferencz, O., Whistler-mode propagation: Solution in homogeneous and weakly inhomogeneous lossy plasmas, Periodica Polytechnica Ser. El. Eng., 38, 267-285, 1994.

Guthart, H., Nose whistler dispersion as a measure of Magnetosphere Electron Temperature, Radio Sci. J. of Res., NBS/USNC-URSI, 69D, 14171424, 1965.

Hasegawa, M., M. Hayakawa, and J. Ohtsu, On the conditions of duct 
trapping of low latitude whistlers, Ann. Geophys., 34, 317, 1978.

Hayakawa, M., Y. Tanaka, K. Ohta, and T. Okada. Absolute intensity of day time whistlers at low and middle latitudes and its latitudinal variation, $J$. Geophys. Res., 59, 67-72, 1986.

Helliwell, R. A. Whistlers and Related Ionospheric Phenomena, Stanford University Press, Stanford, California, 1965.

Inan, U. S., W. A. Sampson, and Y. N. Taranenko, Space-time structure of optical flashes and ionization changes produced by lightning-EMP, Geophys. Res. L., 23, 133-136, 1996.

Itonaga, M., A. Yoshikawa, and K. Yumoto, One-dimensional transient response of the inner magnetosphere at the magnetic equator, 1. Transfer function and poles, J. Geomag. Geoelctr., 49, 21-48, 1997a.

Itonaga, M., A. Yoshikawa, and K. Yumoto, One-dimensional transient response of the inner magnetosphere at the magnetic equator, 2. Analysis of waveforms, J. Geomag. Geoelctr., 49, 49-68, 1997b.

Kamke, E., Differentialgleichungen, Lösungsmethoden and Lösungen I, (Akademische Verlagsgesellschaft, Geest and Porting K. G., Leipzig, (Punkt 23.5), 412-415, 1956.

Kamke, E., Differentialgleichungen, Lösungsmethoden and Lösungen II, (Akademische Verlagsgesellschaft, Geest and Porting K. G., Leipzig, (Punkt 11.1.), 93-98, 1965.

Liang, B. X., T. Bao, and J. S. Xu, Propagation characteristics of nighttime whistlers in the region of equatorial anomaly, J. Atmos. Terr. Phys., 47, 999, 1985.

Nagano, I., S. Yagitani, K. Miyamura, and S. Makino, Full-wave analysis of elves created by lightning-generated electromagnetic pulses, J. Atmos. Terr. Phys., 65, 615-625, 2003.

Ohta, K., T. Tomomatsu, O. Takahashi, and M. Hayakawa, Propagation characteristics of very low latitude whistlers by ray-tracing, IEICE Trans. Commun., J75-B-II, 309, 1992 (in Japanese).

Ohta, K., Y. Nishimura, and T. Kitagawa, Study of propagation character- istics of very low latitude whistlers by means of three-dimensional raytracing computations, J. Geophys. Res., 102, 7537-7546, 1997.

Park, C. G., Methods of determining electron concentration in the magnetosphere from nose whistlers, Tech. Report no. 3454, Radioscience Lab., Stanford University, Stanford, California, 11-17, 1972.

Rowland, H. L., R. F. Fernsler, J. D. Huba, and P. A. Bernhardt, Lightning driven EMP in the upper atmosphere, Geophys. Res. L., 22, 361-364, 1995.

Sazhin, S. S., M. Hayakawa, and K. Bullough, Whistler diagnostics of magnetospheric parameter, A review, Ann. Geophys., 10, 293-308, 1992. Singh, B. and B. A. P. Tantry, On ducting of whistlers at low latitudes, Ann. Geophys., 29, 561-568, 1973.

Singh, R. P., Whistler studies at low latitudes: A review, I. J. Rad. Phys., 22, 139-155, 1993.

Singh, R. P., Lalmani, and U. P. Singh, Electron density distribution derived from low latitude whistler studies, Ann. Geophys., 11, 1011-1017, 1993.

Singh, R. P., A. K. Singh, and D. K. Singh, Plasmaspheric parameters as determined from whistler spectrograms, A review, J. Atmos. Solar Terr. Phys., 60, 495-508, 1998.

Somayajulu, V. V., M. Rao, and B. A. P. Tantry, Whistlers at low latitude, Ind. J. Rad. Space Phys., 1, 102-118, 1972.

Thomson, N. R., Raytracing the paths of very low latitude man-made whistler-mode signals, J. Atmos. Terr. Phys., 49, 321, 1987.

Veronis, G., V. P. Pasko, and U. S. Inan, Characteristics pf mesospheric optical emissions produced by lightning discharges, J. Geophys. Res., 104, 12645-12656, 1999.

K. Singh (e-mail: singh_kalpana@sify.com), R. P. Singh, and O. E. 